\title{
Performance Analysis of Two-Way Relaying with Non-Coherent Differential Modulation
}

\author{
Wei Guan and K. J. Ray Liu, Fellow, IEEE
}

\begin{abstract}
This work focuses on a two-way denoise-andforward relaying system using non-coherent Differential Binary Phase-Shift Keying (DBPSK) modulation, which has the welldefined relay denoising function when channel state information is unknown. We first design the relay denoising function and source decoders using Maximum Likelihood (ML) principles for the general case with $K$ parallel relays. As the ML denoising function is hard to manipulate, we approximate it as a multi-user detector followed by a physical layer network coding encoder and obtain the closed-form relay decoding error. For the singlerelay case, we show that the ML source decoder is actually equivalent to the typical DBPSK decoder for the relay-source channel and thus derive the exact end-to-end Bit Error Rate (BER). To minimize the average BER, we also investigate the power allocation problem by use of asymptotic analysis at high Signal-to-Noise Ratio (SNR). We show that the optimal source power is inversely proportional to the square root of the channel gain of the source-relay channel, and the optimal relay power decreases with SNR. For the multi-relay case, though the exact analysis is intractable, we develop upper bound and lower bound on BER and show that the diversity order is exactly $\left\lceil\frac{K}{2}\right\rceil$.
\end{abstract}

Index Terms-Two-way relaying, differential modulation, BER, diversity order, power allocation.

\section{INTRODUCTION}

W Ireless channel has negative effects on signal propagation in terms of channel fading and path loss, and cooperative communications, which is assisted by a set of fixed or mobile relay nodes, can elegantly overcome these shortcomings by providing distributed spatial diversity and making a more efficient use of transmit power [1]. As the terminals generally cannot transmit and receive on the same channel simultaneously due to hardware limitations, most of the recent literatures focus on half-duplex relaying protocol, such as Amplify-and-Forward (AF) and Decode-and-Forward (DF) [2][3]. The DF relays first decode the source information and then forward a re-encoded signal, while the AF relays just amplify the receive signals subject to relay power constraints. However, the above half-duplex relaying protocol will inevitably reduce the channel use. This is because both of the AF and DF relays use two time phases to deliver only one information unit, which introduces a pre-log factor $\frac{1}{2}$ on the spectral efficiency [4]. The traditional selective relaying protocol [2][5] can partially recover such rate loss, as the relay nodes are active only when necessary and thus

Manuscript received October 11, 2010; revised April 5, 2011; accepted April 28, 2011. The associate editor coordinating the review of this paper and approving it for publication was R. Nabar.

W. Guan and K. J. Ray Liu are with the Department of Electrical and Computer Engineering, University of Maryland, College Park, MD 20742, USA (e-mail: \{wguan, kjrliu\}@umd.edu).

Digital Object Identifier 10.1109/TWC.2011.11.101798 save the redundant channel use. More recently, Two-Way Relaying (TWR), where the two source nodes exchange their information at the same time with the help of the relay nodes, has drawn lots of attention due to its potential to fully recover the rate loss resulted from half-duplexing. There are generally two kinds of TWR protocols depending on the number of used time phases, i.e., Two-Phase TWR (2P-TWR) and Three-Phase TWR (3P-TWR).

In 3P-TWR, two source nodes send information to the relays successively over the first two phases, and the relays broadcast a mixture of the received signal during the third Broadcasting (BC) phase. The 3P-DF-TWR is first proposed in [6] for the single-relay case, where the relays perform Network Coding (NC) [7] at bit-level through exclusive-or operations. $\mathrm{NC}$ is power efficient in that the relays only need to send a single symbol, based on which both sources can uniquely decode the information from the other end by use of its own side information. It also shows there the 3P-DF-TWR can achieve a maximum throughput gain of $\frac{1}{3}$ over the traditional one-way relaying that requires a total of 4 phases to complete the same information exchange. In the 3P-AF-TWR proposed in [8], the relay forwards a weighted sum of the signals received in the first two phases. By properly choosing the weights, lower Bit Error Rate (BER) can be achieved than the traditional AF relaying.

The 2P-TWR makes one more step toward channel use savings by letting the two sources transmit simultaneously in a single Multiple Access (MA) phase. Early work on 2PAF-TWR and 2P-DF-TWR can be found in [4], which shows great enhancement on sum-rates from an information theoretic viewpoint. However, the proposed joint decode-and-forward protocol is hard to realize in practice without any special multiple access technique, as the source signals already combine in the air and decoding them separately results in large BER. With such concerns, [9] proposes a new Denoise-and-Forward (DNF) protocol, where the relays apply a denoising function to map the receive signal into another quantized symbol that can be used by each source node to uniquely decode the symbol transmitted from the other end. A similar scheme called Physical-Layer Network Coding (PLNC) is proposed in [10], where the condition to guarantee one-to-one mapping between NC and PLNC is also given. Later work [11] shows that 2P-DNF-TWR has higher sum-rates than 2P-AF-TWR and 2P-DF-TWR; [12] derives the closed-form BER of 2PDNF-TWR with coherent BPSK modulation; and [8] and [13] reveal that 2P-TWR generally has higher sum-rates, whereas 3P-TWR enjoys lower BER instead.

While TWR opens a door to improve spectral efficiency, most of the existing work [8], [9] and [11]-[13] all assume 
that the terminals have full knowledge of Channel State Information (CSI), which on the other hand is hard to acquire in a fast-fading environment [3]. Such concerns motivate the non-coherent modulation schemes, which have been widely examined for the traditional one-way relaying. For example, [14] develops the Maximum Likelihood (ML) decoders for DF relaying with non-coherent binary frequency-shift keying modulation and shows that the diversity order is roughly half of the number of relays; for single-relay case, [15] designs the selective relaying protocol and analyzes the corresponding BER; finally, [16]-[18] propose relay selection methods for the multi-relay case and show that the full diversity order can be achieved. In a limited number of literatures about TWR using non-coherent modulation, [19] designs the non-coherent decoder for minimum-shift keying signals and validates the throughput gain on a software radio testbed; [20] gives the symbol error rate for 2P-AF-TWR with relay selection; and [21] designs a set of non-coherent decoders for both 2P-AFTWR and 2P-DNF-TWR using differential modulation, .

As summarized above, 2P-DNF-TWR with non-coherent modulation can benefit from both the high spectral efficiency and reduced channel estimation overhead. However, early work on DNF protocol focuses mainly on AWGN channels [9][10]. Although the decoder design issue in the fading channel has been partly addressed in [21] for the single-relay system, the performance is mainly evaluated through simulation and there are no discussions about resource allocation. The adaptive denoising function design in fading channels is recently given in [22] where perfect knowledge about CSI is assumed. When CSI is unknown, how to optimize the denoising function is still an open problem except for the special case with BPSK modulation. Indeed, a thorough investigation into such particular scheme could not only shed some light on the denoising function design for the more general system using higher-order non-coherent modulations, but also help to resolve other wireless network design problems like relay deployment and resource allocations, and such concerns motivate the current work.

Specifically, we focus on a 2P-DNF-TWR system using non-coherent Differential BPSK (DBPSK) modulation with $K$ parallel relays. We first derive the relay denoising function and source decoder using ML principles, and then proceed to analyze the corresponding decoding error at each terminal. As it is hard to manipulate the ML denoising function directly, we approximate it as a Multi-User Detector (MUD) followed by a PLNC encoder and obtain the closed-form relay decoding error. For the single-relay case, we reveal the equivalence between the ML source decoder and the typical DBPSK decoder for the relay-source channel, based on which we obtain the exact end-to-end BER. We further investigate the power allocation problem so as to minimize the average system BER by use of asymptotic analysis, and show that the optimal source power is inversely proportional to the square root of the channel gain of the source-relay channel, and the optimal relay power decreases with Signal-to-Noise Ratio (SNR). For the multi-relay case, though the exact analysis is intractable, we develop upper bound and lower bound on BER and show that the diversity order is exactly $\left\lceil\frac{K}{2}\right\rceil$. We validate all our results by computer simulations.

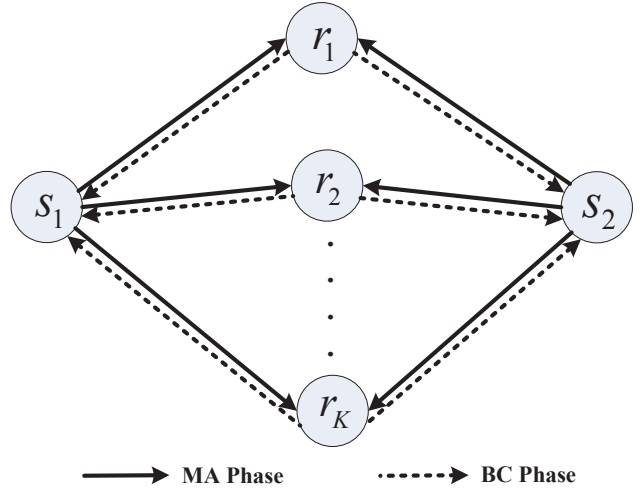

Fig. 1. System model of 2P-DNF-TWR.

The rest of this paper is organized as follows: In Section II, we describe the system model and design the relay denoising function and source decoders. For single-relay case, exact error performance is given in Section III, where we also formulate the power allocation problem. Then in Section IV we analyze the diversity order of a multi-relay system, and we provide simulation results in Section V. Finally some conclusions are given in Section VI.

Notations: Boldface lowercase letter a and boldface uppercase letter $\mathbf{A}$ represent vector in column form and matrix, respectively. $\|\mathbf{a}\|$ and $|\mathbf{A}|$ represent the Euclidean norm of a vector a and the determinant of a square matrix $\mathbf{A}$, respectively. $(\cdot)^{*},(\cdot)^{T}$ and $(\cdot)^{H}$ stand for conjugate, transpose and conjugate transpose, respectively. We shall use abbreviation i.i.d. for independent and identically distributed, and denote $Z \sim \mathcal{C N}\left(\mu, \sigma^{2}\right)$ as a circularly symmetric complex Gaussian random variable $Z$ with i.i.d. real part and imaginary part $\sim \mathcal{N}\left(\mu, \frac{\sigma^{2}}{2}\right)$. We define $\operatorname{sign}(x)=1$ if $x>0$ and 0 otherwise. Finally, the probability of an event $\mathcal{A}$ and the Probability Density Function (PDF) of a random variable $Z$ are denoted by $P(\mathcal{A})$ and $f(Z)$, respectively.

\section{System Model}

Consider a narrow-band 2P-DNF-TWR system shown in Fig. 1, where two sources $S_{1}$ and $S_{2}$ want to exchange information with the help of $K$ parallel relays. At the beginning of the MA phase, the $i$ th $(i=1,2)$ source first generates a sequence of i.i.d uncoded BPSK symbols $b_{i}(n) \in\{-1,1\}$ of length $L$, where $n=1,2, \ldots, L$ is the symbol index. These raw symbols are then re-encoded through differential modulation, i.e., $x_{i}(n)=x_{i}(n-1) \times b_{i}(n)$ for $n=1,2, \ldots, L$ with $x_{i}(0)=1$ being the reference symbol. The two sources then send the whole block of differentially encoded symbols simultaneously to all the relays during MA phase. To facilitate demonstrations, we define a sequence of auxiliary symbols $b(n)=b_{1}(n) \times b_{2}(n) \in\{-1,1\}$ for $n=1,2, \ldots, L$ to indicate whether the two raw BPSK symbols have the same signs or not. Note that because each source knows its own symbol, this common information $b(n)$ is sufficient for both sources to decode the symbol from the other end.

At the end of MA phase, the $n$th $(n=0,1, \ldots, L)$ symbol 


$$
\left\{\begin{array}{l}
\boldsymbol{\Sigma}_{b_{1}(n)=1, b_{2}(n)=1}^{k} \triangleq \boldsymbol{\Sigma}_{1, r_{k}}=N_{0}\left(\gamma_{1, k}+\gamma_{2, k}+1\right) \mathbf{I}_{2}+N_{0}\left(\gamma_{1, k}+\gamma_{2, k}\right) \hat{\mathbf{I}}_{2} \\
\boldsymbol{\Sigma}_{b_{1}(n)=-1, b_{2}(n)=-1}^{k} \boldsymbol{\Sigma}_{2, r_{k}}=N_{0}\left(\gamma_{1, k}+\gamma_{2, k}+1\right) \mathbf{I}_{2}-N_{0}\left(\gamma_{1, k}+\gamma_{2, k}\right) \hat{\mathbf{I}}_{2} \\
\boldsymbol{\Sigma}_{b_{1}(n)=1, b_{2}(n)=-1}^{k} \triangleq \boldsymbol{\Sigma}_{3, r_{k}}=N_{0}\left(\gamma_{1, k}+\gamma_{2, k}+1\right) \mathbf{I}_{2}+N_{0}\left(\gamma_{1, k}-\gamma_{2, k}\right) \hat{\mathbf{I}}_{2} \\
\boldsymbol{\Sigma}_{b_{1}(n)=-1, b_{2}(n)=1}^{k} \triangleq \boldsymbol{\Sigma}_{4, r_{k}}=N_{0}\left(\gamma_{1, k}+\gamma_{2, k}+1\right) \mathbf{I}_{2}+N_{0}\left(\gamma_{2, k}-\gamma_{1, k}\right) \hat{\mathbf{I}}_{2}
\end{array}\right.
$$

received at the $k$ th $(k=1,2, \ldots, K)$ relay is then

$$
y_{k}(n)=\sqrt{P_{s_{1}}} h_{1, k}^{M A} x_{1}(n)+\sqrt{P_{s_{2}}} h_{2, k}^{M A} x_{2}(n)+w_{k}^{M A}(n),
$$

where $P_{s_{i}}=\alpha_{i} P$ is the $i$ th $(i=1,2)$ source power, $P$ is the total power and $\alpha_{i} \in[0,1]$ stands for the corresponding source power ratio. $h_{i, k}^{M A} \sim \mathcal{C N}\left(0, \sigma_{i, k}^{2}\right)$ is the independent channel coefficient from the $i$ th $(i=1,2)$ source to the $k$ th $(k=1,2, \ldots, K)$ relay during MA phase, where $\sigma_{i, k}^{2}$ is the channel gain. Here we assume that the channels remain unchanged within one block of length $(L+1)^{1}$; however, no terminals know such CSI so as to eliminate the channel estimation overhead. Finally, $w_{k}^{M A}(n) \sim \mathcal{C N}\left(0, N_{0}\right)$ is the independent Additive White Gaussian Noise (AWGN) at the $k$ th $(k=1,2, \ldots, K)$ relay within the $n$th $(n=0,1, \ldots, L)$ symbol interval during MA phase.

With DNF protocol[9][10], the $k$ th $(k=1,2, \ldots, K)$ relay just maps the $n$th $(n=1,2 \ldots, L)$ receive symbol to another BPSK symbol $\hat{b}_{r_{k}}(n) \in\{-1,1\}$ that can be used by each source to uniquely decode the symbol transmitted from the other end. Here $\hat{b}_{r_{k}}(n) \in\{-1,1\}$ can be regarded as an estimate of the auxiliary symbol $b(n)$, so the relay denoising function is actually equivalent to the decoder for $b(n)$. As no CSI is available, we use the single-symbol ML decoder ${ }^{2}$ similar to that proposed in [21] throughout this work, i.e.,

$$
\hat{b}_{r_{k}}(n)=\arg \max _{b(n) \in\{-1,1\}} f\left(\mathbf{y}_{k}(n) \mid b(n)\right),
$$

where $\mathbf{y}_{k}(n)=\left(y_{k}(n), y_{k}(n-1)\right)^{T}$ is the vector of two consecutive receive symbols. It is easy to show that given $b_{1}(n)$ and $b_{2}(n),\left.\mathbf{y}_{k}(n)\right|_{b_{1}(n), b_{2}(n)} \sim \mathcal{C N}\left(\mathbf{0}, \boldsymbol{\Sigma}_{b_{1}(n), b_{2}(n)}^{k}\right)$, where the conditional covariance matrices are given by (3) on the top of this page. Here $\gamma_{i, k}=\frac{P_{s_{i}} \sigma_{i, k}^{2}}{N_{0}}=\alpha_{i} \sigma_{i, k}^{2} \gamma$ is the channel SNR from the $i$ th $(i=1,2)$ source to the $k$ th $(k=1,2, \ldots, K)$ relay, $\gamma=\frac{P}{N_{0}}$ is the system $\mathrm{SNR}$, and

$$
\mathbf{I}_{2}=\left(\begin{array}{ll}
1 & 0 \\
0 & 1
\end{array}\right), \hat{\mathbf{I}}_{2}=\left(\begin{array}{ll}
0 & 1 \\
1 & 0
\end{array}\right)
$$

are two constant matrices. Based on the law of total probability, the conditional PDF of $\mathbf{y}_{k}(n)$ can be expressed as

$$
f\left(\mathbf{y}_{k}(n) \mid b(n)\right)=\frac{1}{2} \sum_{b_{1}(n) \times b_{2}(n)=b(n)} f\left(\mathbf{y}_{k}(n) \mid b_{1}(n), b_{2}(n)\right) .
$$

After some manipulations, we can re-write the ML decoder (2) as

$$
\hat{b}_{r_{k}}(n)=\operatorname{sign}\left(\ln \left(\operatorname{lrf}\left(\mathbf{y}_{k}(n) \mid b(n)\right)\right)\right),
$$

\footnotetext{
${ }^{1}$ For the following single-symbol decoding, we only require that the channels keep unchanged within the interval of two consecutive symbols. The quasi-static assumption is just to simplify the notations.

${ }^{2}$ Actually $b(n)$ is uniformly distributed. So the ML decoder is equivalent to the maximum a posterior decoder. The same argument holds for the following source decoder.
}

where

$$
\operatorname{lrf}\left(\mathbf{y}_{k}(n) \mid b(n)\right)=\frac{g\left(\mathbf{y}_{k}(n), \boldsymbol{\Sigma}_{1, r_{k}}\right)+g\left(\mathbf{y}_{k}(n), \boldsymbol{\Sigma}_{2, r_{k}}\right)}{g\left(\mathbf{y}_{k}(n), \boldsymbol{\Sigma}_{3, r_{k}}\right)+g\left(\mathbf{y}_{k}(n), \boldsymbol{\Sigma}_{4, r_{k}}\right)}
$$

is the Likelihood Ratio Function (LRF) of $\mathbf{y}_{k}(n)$ conditioned on $b(n)$, and

$$
g(\mathbf{y}, \boldsymbol{\Sigma})=\frac{1}{\pi^{2}|\boldsymbol{\Sigma}|} \exp \left(-\mathbf{y}^{H} \boldsymbol{\Sigma}^{-1} \mathbf{y}\right)
$$

is the PDF of $\mathbf{y} \sim \mathcal{C N}(\mathbf{0}, \boldsymbol{\Sigma})$. After decoding, the $k$ th $(k=1,2 \ldots, K)$ relay re-encode $\left\{\hat{b}_{r_{k}}(n)\right\}_{n=1}^{L}$ into $t_{k}(n)=t_{k}(n-1) \times \hat{b}_{r_{k}}(n)$ for $n=1,2, \ldots, L$ through differential modulation with $t_{k}(0)=0$ being the reference symbol.

During BC phase, all relays broadcast their own differentially re-encoded symbols together through a set of orthogonal channels. It is worth noting that as the relays have no CSI, the typical transmit diversity technique is unavailable here. So the co-channel relaying will not bring any diversity gain compared to the single-relay case, as the broadcast signals would be randomly combined in the air. In practice, the orthogonal relaying can happen in a fixed relay system where all relays operate on its own dedicated channel. At the end of BC phase, the $i$ th $(i=1,2)$ source will receive from the $k$ th $(k=1,2 \ldots, K)$ relay

$$
r_{k, i}(n)=\sqrt{P_{r_{k}}} h_{k, i}^{B C} t_{k}(n)+w_{k, i}^{B C}(n), n=0,1, \ldots, L,
$$

where $P_{r_{k}}=\beta_{k} P$ is the $k$ th $(k=1,2 \ldots, K)$ relay power and $\beta_{k} \in[0,1]$ stands for the corresponding relay power ratio. $h_{k, i}^{B C} \sim \mathcal{C N}\left(0, \sigma_{i, k}^{2}\right)$ is the independent channel coefficient from the $k$ th $(k=1,2 \ldots, K)$ relay to the $i$ th $(i=1,2)$ source during BC phase, and we assume $h_{k, i}^{B C}$ and $h_{i, k}^{M A}$ are independent but have the same channel gain, which is determined by the distance between two terminals. However, all the results in this work can be easily extended to the more general case with different channel gains. Finally, $w_{k, i}^{B C}(n) \sim \mathcal{C N}\left(0, N_{0}\right)$ is the independent AWGN on the $k$ th $(k=1,2, \ldots, K)$ relaysource channel at the $i$ th $(i=1,2)$ source within the $n$th $(n=0,1, \ldots, L)$ symbol interval during $\mathrm{BC}$ phase.

As explained before, each source only needs to detect $b(n)$ so as to decode the symbol from the other end. For example, if the decoded symbol for $b(n)$ at source 1 is $\hat{b}_{s_{1}}(n)=1$, then $b_{2}(n)$ can be decoded as $\hat{b}_{2, s_{1}}(n)=b_{1}(n)$, otherwise $\hat{b}_{2, s_{1}}(n)=-b_{1}(n)$ if $\hat{b}_{s_{1}}(n)=-1$. Again we assume the $i$ th $(i=1,2)$ source uses the single-symbol ML decoder based on the observations $\left\{\mathbf{r}_{k, i}(n)\right\}_{k=1}^{K}$, i.e.,

$$
\hat{b}_{s_{i}}(n)=\arg \max _{b(n) \in\{-1,1\}} f\left(\left\{\mathbf{r}_{k, i}(n)\right\}_{k=1}^{K} \mid b(n)\right),
$$

where $\mathbf{r}_{k, i}(n)=\left(r_{k, i}(n), r_{k, i}(n-1)\right)^{T}$ is the vector of two consecutive receive symbols from the $k$ th $(k=1,2, \ldots, K)$ 


$$
f\left(\left\{\mathbf{r}_{k, i}(n)\right\}_{k=1}^{K} \mid b(n)\right)=\prod_{k=1}^{K} \sum_{\hat{b}_{r_{k}}(n) \in\{-1,1\}} f\left(\mathbf{r}_{k, i}(n) \mid \hat{b}_{r_{k}}(n)\right) P\left(\hat{b}_{r_{k}}(n) \mid b(n)\right)
$$

$$
\operatorname{lrf}\left(\mathbf{r}_{k, i}(n) \mid b(n)\right)=\frac{g\left(\mathbf{r}_{k, i}(n), \boldsymbol{\Sigma}_{1, s_{i}}^{k}\right)\left(1-P_{M, r_{k}}\right)+g\left(\mathbf{r}_{k, i}(n), \boldsymbol{\Sigma}_{2, s_{i}}^{k}\right) P_{M, r_{k}}}{g\left(\mathbf{r}_{k, i}(n), \boldsymbol{\Sigma}_{1, s_{i}}^{k}\right) P_{F, r_{k}}+g\left(\mathbf{r}_{k, i}(n), \boldsymbol{\Sigma}_{2, s_{i}}^{k}\right)\left(1-P_{F, r_{k}}\right)}
$$

$$
\operatorname{lrf}\left(\mathbf{y}_{k}(n) \mid b(n)\right)=\frac{\left|\boldsymbol{\Sigma}_{3, r_{k}}\right|}{\left|\boldsymbol{\Sigma}_{1, r_{k}}\right|} \frac{\cosh \left(\frac{N_{0}\left(\gamma_{1, k}+\gamma_{2, k}\right)}{\boldsymbol{\Sigma}_{1, r_{k}} \mid} \mathbf{y}_{k}^{H}(n) \hat{\mathbf{I}}_{2} \mathbf{y}_{k}(n)\right)}{\cosh \left(\frac{N_{0}\left(\gamma_{1, k}-\gamma_{2, k}\right)}{\left|\boldsymbol{\Sigma}_{3, r_{k}}\right|} \mathbf{y}_{k}^{H}(n) \hat{\mathbf{I}}_{2} \mathbf{y}_{k}(n)\right)} \exp \left(\frac{\left|\boldsymbol{\Sigma}_{1, r_{k}}\right|-\left|\boldsymbol{\Sigma}_{3, r_{k}}\right|}{\left|\boldsymbol{\Sigma}_{1, r_{k}}\right|\left|\boldsymbol{\Sigma}_{3, r_{k}}\right|} N_{0}\left(\gamma_{1, k}+\gamma_{2, k}+1\right)\left\|\mathbf{y}_{k}(n)\right\|^{2}\right)
$$

relay, and $\left.\mathbf{r}_{k, i}(n)\right|_{\hat{b}_{r_{k}}(n)} \sim \mathcal{C N}\left(\mathbf{0}, \boldsymbol{\Sigma}_{\hat{b}_{r_{k}}(n), s_{i}}^{k}\right)$ where the conditional covariance matrices are given by

$$
\left\{\begin{array}{l}
\boldsymbol{\Sigma}_{\hat{b}_{r_{k}}(n)=1, s_{i}}^{k} \triangleq \boldsymbol{\Sigma}_{1, s_{i}}^{k}=N_{0}\left(\bar{\gamma}_{k, i}+1\right) \mathbf{I}_{2}+N_{0} \bar{\gamma}_{k, i} \hat{\mathbf{I}}_{2} \\
\boldsymbol{\Sigma}_{\hat{b}_{r_{k}}(n)=-1, s_{i}}^{k} \triangleq \Sigma_{2, s_{i}}^{k}=N_{0}\left(\bar{\gamma}_{k, i}+1\right) \mathbf{I}_{2}-N_{0} \bar{\gamma}_{k, i} \hat{\mathbf{I}}_{2}
\end{array},\right.
$$

where $\bar{\gamma}_{k, i}=\frac{P_{r_{k}} \sigma_{i, k}^{2}}{N_{0}}=\beta_{k} \sigma_{i, k}^{2} \gamma$ is the channel SNR from the $k$ th $(k=1,2, \ldots, K)$ relay to the $i$ th $(i=1,2)$ source. As the signals from different relays are independent conditioned on $b(n)$, we can rewrite the joint PDF in (9) as (11) given on the top of next page, where we use the law of total probability and the fact $\mathbf{r}_{k, i}(n)$ is independent with $b(n)$ conditioned on $\hat{b}_{r_{k}}(n)$. Based on (11), the ML source decoder (9) can be simplified to

$$
\hat{b}_{s_{i}}(n)=\operatorname{sign}\left(\sum_{k=1}^{K} \ln \left(\operatorname{lrf}\left(\mathbf{r}_{k, i}(n) \mid b(n)\right)\right)\right),
$$

where $\operatorname{lr} f\left(\mathbf{r}_{k, i}(n) \mid b(n)\right)$ given on the top of this page is the LRF of $\mathbf{r}_{k, i}(n)$ conditioned on $b(n)$, and

$$
\begin{aligned}
P_{M, r_{k}} & =P\left(\hat{b}_{r_{k}}(n)=-1 \mid b(n)=1\right) \\
& =P\left(\operatorname{lrf}\left(\mathbf{y}_{k}(n) \mid b(n)\right) \leq 1 \mid b(n)=1\right), \\
P_{F, r_{k}} & =P\left(\hat{b}_{r_{k}}(n)=1 \mid b(n)=-1\right) \\
& =P\left(\operatorname{lrf}\left(\mathbf{y}_{k}(n) \mid b(n)\right)>1 \mid b(n)=-1\right)
\end{aligned}
$$

are two kinds of conditional decoding error at the $k$ th $(k=1,2, \ldots, K)$ relay. The calculation of these two terms is postponed to the next section. Note that as both the relay decoder (2) and source decoder (9) depend only on the secondorder statistics of all channels, which remain unchanged over time, the whole system can benefit from a great reduction on channel estimation overheads.

\section{Performance Analysis: Single-Relay Case}

In this section, we will examine the error performance of the proposed relay decoder (2) and source decoder (9) for the single-relay case. Without loss of generality, we assume only the $k$ th $(k \in\{1,2, \ldots, K\})$ relay is activated to assist the information exchange between two sources. To optimize the end-to-end error performance, we shall also investigate the power allocation problem.

\section{A. Relay Decoding Error}

By use of the law of total probability, we can write the relay decoding error as

$$
P\left(\hat{b}_{r_{k}}(n) \neq b(n)\right) \triangleq P_{e, r_{k}}=\frac{P_{M, r_{k}}+P_{F, r_{k}}}{2},
$$

where $P_{M, r_{k}}$ and $P_{F, r_{k}}$ are two kinds of conditional decoding error defined in (14) and (15), and both of them are related with $\operatorname{lr} f\left(\mathbf{y}_{k}(n) \mid b(n)\right)$. After substituting (7) into (6) and doing some manipulations, we have (17) on the top of this page, where $\cosh (x)=\frac{e^{x}+e^{-x}}{2}$ is the hyperbolic cosine function. As it is really hard to analyze the error probability based on the above LRF, we use the following approximation to facilitate the analysis

$$
\cosh (x) \approx \frac{\max \left(e^{x}, e^{-x}\right)}{2}=\frac{e^{|x|}}{2},
$$

which is quite tight when $|x|$ is not too small. After such approximation, only exponential terms are left with the exponent being a quadratic form of $\mathbf{y}_{k}(n)$, which is analytically tractable.

After substituting (18) back into (17), we will arrive at

$$
\operatorname{lrf}\left(\mathbf{y}_{k}(n) \mid b(n)\right) \approx \frac{\max \left(g\left(\mathbf{y}_{k}(n), \boldsymbol{\Sigma}_{1, r_{1}}\right), g\left(\mathbf{y}_{k}(n), \boldsymbol{\Sigma}_{2, r_{k}}\right)\right)}{\max \left(g\left(\mathbf{y}_{k}(n), \boldsymbol{\Sigma}_{3, r_{k}}\right), g\left(\mathbf{y}_{k}(n), \boldsymbol{\Sigma}_{4, r_{k}}\right)\right)} .
$$

Now if we use (19) instead in (5), it is easy to see that this suboptimal decoder is actually a MUD

$$
\begin{aligned}
& \left(\hat{b}_{1, r_{k}}(n), \hat{b}_{2, r_{k}}(n)\right) \\
& \quad=\arg \max _{b_{i}(n) \in\{-1,1\}, i=1,2} f\left(\mathbf{y}_{k}(n) \mid b_{1}(n), b_{2}(n)\right)
\end{aligned}
$$

followed by a PLNC encoder $\hat{b}_{r_{k}}(n)=\hat{b}_{1, r_{k}}(n) \times \hat{b}_{2, r_{k}}(n)$. That is, the relay first jointly decodes the BPSK symbols $b_{1}(n)$ and $b_{2}(n)$, and then maps the decoded symbols to a single BPSK symbol $\hat{b}_{r_{k}}(n)$ as an estimate of the indicator symbol $b(n)$. As we shall see in the simulation section, this suboptimal relay decoder works almost as well as the ML decoder (5) in all cases. The reason is that the two PDFs of $\left(b_{1}(n), b_{2}(n)\right)$ corresponding to the same $b(n)$ are actually well separated. As a result, the ML region of $b(n)$ is very close to the direct union of the individual ML regions of $\left(b_{1}(n), b_{2}(n)\right)$, which leads to the max operation in (19). 


$$
\begin{array}{rl}
P_{M, r_{k}}=\frac{1}{2} \sum_{b_{1}(n) \times b_{2}(n)=1} & P\left(\left(a_{k}+b_{k}\right)\left|\hat{y}_{k, 1}(n)\right|^{2}+\left(a_{k}-b_{k}\right)\left|\hat{y}_{k, 2}(n)\right|^{2} \leq \ln \gamma_{t h}^{k} \&\right. \\
& \left.\left(a_{k}-b_{k}\right)\left|\hat{y}_{k, 1}(n)\right|^{2}+\left(a_{k}+b_{k}\right)\left|\hat{y}_{k, 2}(n)\right|^{2} \leq \ln \gamma_{t h}^{k} \mid b_{1}(n), b_{2}(n)\right)
\end{array}
$$

$$
b_{k}=\frac{4 \gamma_{1, k} \gamma_{2, k}\left(\gamma_{1, k}+\gamma_{2, k}\right)+2 \min \left(\gamma_{1, k}, \gamma_{2, k}\right)\left(2 \gamma_{1, k}+2 \gamma_{2, k}+1\right)}{N_{0}\left(2 \gamma_{1, k}+2 \gamma_{2, k}+1\right)\left(2 \gamma_{1, k}+1\right)\left(2 \gamma_{2, k}+1\right)}
$$

$$
h\left(t_{1}, t_{2}, a, b, \gamma\right)=\frac{4 a b t_{1} t_{2}}{a^{2}\left(t_{1}-t_{2}\right)^{2}-b^{2}\left(t_{1}+t_{2}\right)^{2}} \exp \left(-\frac{t_{1}+t_{2}}{2 a} \ln \gamma\right)
$$

To characterize the error performance, let us first calculate $P_{M, r_{k}}$. After substituting (19) into (14) and making some manipulations, we have (21) on the top of this page, where

$$
\begin{gathered}
a_{k}=-\frac{4 \gamma_{1, k} \gamma_{2, k}\left(\gamma_{1, k}+\gamma_{2, k}+1\right)}{N_{0}\left(2 \gamma_{1, k}+2 \gamma_{2, k}+1\right)\left(2 \gamma_{1, k}+1\right)\left(2 \gamma_{2, k}+1\right)}, \\
\gamma_{t h}^{k}=\frac{\left(2 \gamma_{1, k}+2 \gamma_{2, k}+1\right)}{\left(2 \gamma_{1, k}+1\right)\left(2 \gamma_{2, k}+1\right)},
\end{gathered}
$$

and $b_{k}$ is given in (23) on the top of this page, and we define

$$
\hat{\mathbf{y}}_{k}(n)=\left(\hat{y}_{k, 1}(n), \hat{y}_{k, 2}(n)\right)^{T}=\frac{1}{\sqrt{2}}\left(\begin{array}{cc}
1 & 1 \\
1 & -1
\end{array}\right) \mathbf{y}_{k}(n)
$$

as an auxiliary random vector. Since $\left.\mathbf{y}_{k}(n)\right|_{b_{1}(n), b_{2}(n)} \sim \mathcal{C N}\left(\mathbf{0}, \boldsymbol{\Sigma}_{b_{1}(n), b_{2}(n)}^{k}\right), \quad\left|\hat{y}_{k, 1}(n)\right|^{2} \quad$ and $\left|\hat{y}_{k, 2}(n)\right|^{2}$ are actually independent exponential random variables conditioned on $b_{1}(n)$ and $b_{2}(n)$. Therefore, (20) can be easily measured as

$$
P_{M, r_{k}}=h\left(u_{1, k}, u_{2, k}, a_{k}, b_{k}, \gamma_{t h}^{k}\right),
$$

where $h\left(t_{1}, t_{2}, a, b, \gamma\right)$ given in (27) on the top of this page is a function with five parameters, and

$$
u_{1, k}=\frac{1}{N_{0}\left(2 \gamma_{1, k}+2 \gamma_{2, k}+1\right)}, u_{2, k}=\frac{1}{N_{0}} .
$$

In a similar manner, we can show that

$$
P_{F, r_{k}}=1-h\left(u_{3, k}, u_{4, k}, a_{k}, b_{k}, \gamma_{t h}^{k}\right),
$$

where

$$
u_{3, k}=\frac{1}{N_{0}\left(2 \gamma_{1, k}+1\right)}, u_{4, k}=\frac{1}{N_{0}\left(2 \gamma_{2, k}+1\right)} .
$$

Finally, plugging (26) and (29) back into (16) leads to the closed-form relay decoding error.

\section{B. Source Decoding Error}

When there is only one active relay in the system, the $i$ th $(i=1,2)$ source decoder (12) can be reduced to

$$
\begin{aligned}
\hat{b}_{s_{i}}(n) & =\operatorname{sign}\left(\ln \left(\operatorname{lrf}\left(\mathbf{r}_{k, i}(n) \mid b(n)\right)\right)\right) \\
& =\operatorname{sign}\left(\ln \left(\operatorname{lrf}\left(\mathbf{r}_{k, i}(n) \mid \hat{b}_{r_{k}}(n)\right)\right)\right) \\
& \triangleq \hat{b}_{r_{k}, s_{i}}(n),
\end{aligned}
$$

where

$$
\operatorname{lrf}\left(\mathbf{r}_{k, i}(n) \mid \hat{b}_{r_{k}}(n)\right)=\frac{g\left(\mathbf{r}_{k, i}(n), \boldsymbol{\Sigma}_{1, s_{i}}^{k}\right)}{g\left(\mathbf{r}_{k, i}(n), \boldsymbol{\Sigma}_{2, s_{i}}^{k}\right)}
$$

is the LRF of $\mathbf{r}_{k, i}(n)$ conditioned on $\hat{b}_{r_{k}}(n)$. Note that the decoder on the second line of (31) is actually a typical noncoherent DBPSK decoder [23, Eqn.(14-4-23)] for the pointto-point channel from the $k$ th $(k \in\{1,2, \ldots, K\})$ relay to the $i$ th $(i=1,2)$ source, whose output $\hat{b}_{r_{k}, s_{i}}(n)$ is an estimate of the decoded symbol $\hat{b}_{r_{k}}(n)$ at the corresponding relay. With such equivalence relation at hand, we can write the source decoding error as

$$
\begin{aligned}
P\left(\hat{b}_{s_{i}}(n) \neq b(n)\right) & =P\left(\hat{b}_{r_{k}, s_{i}}(n) \neq b(n)\right) \\
& \triangleq P_{e, s_{i}}^{k}=\frac{1}{2}\left(P_{M, s_{i}}^{k}+P_{F, s_{i}}^{k}\right),
\end{aligned}
$$

where

$$
\begin{aligned}
P_{M, s_{i}}^{k} & =P\left(\hat{b}_{s_{i}}(n)=-1 \mid b(n)=1\right) \\
& =P\left(\hat{b}_{r_{k}, s_{i}}(n)=-1 \mid b(n)=1\right), \\
P_{F, s_{i}}^{k} & =P\left(\hat{b}_{s_{i}}(n)=1 \mid b(n)=-1\right) \\
& =P\left(\hat{b}_{r_{k}, s_{i}}(n)=1 \mid b(n)=-1\right)
\end{aligned}
$$

are two kinds of conditional decoding error at the $i$ th $(i=1,2)$ source, and we use the relation $\hat{b}_{s_{i}}(n)=\hat{b}_{r_{k}, s_{i}}(n)$ in (33)-(35). After expanding (34) by use of the law of total probability, we have (36) on the top of next page, where we use in (a) the fact that $\hat{b}_{r_{k}, s_{i}}(n)$ is independent of $b(n)$ conditioned on $\hat{b}_{r_{k}}(n)$, and in (b) we rely on the fact that the two kinds of conditional decoding error of a typical non-coherent DBPSK decoder are equal and are given by [23, Eqn.(14-4-26)]

$$
\begin{gathered}
P\left(\hat{b}_{r_{k}, s_{i}}(n)=1 \mid \hat{b}_{r_{k}}(n)=-1\right) \\
\quad=P\left(\hat{b}_{r_{k}, s_{i}}(n)=-1 \mid \hat{b}_{r_{k}}(n)=1\right) \\
\triangleq P_{D, s_{i}}^{k}=\frac{1}{2\left(\bar{\gamma}_{k, i}+1\right)} .
\end{gathered}
$$

In a similar way, we can derive

$$
P_{F, s_{i}}^{k}=\left(1-P_{D, s_{i}}^{k}\right) P_{F, r_{k}}+P_{D, s_{i}}^{k}\left(1-P_{F, r_{k}}\right) .
$$

Plugging (36) and (38) back into (33) we have

$$
P_{e, s_{i}}^{k}=\left(1-P_{D, s_{i}}^{k}\right) P_{e, r_{k}}+P_{D, s_{i}}^{k}\left(1-P_{e, r_{k}}\right),
$$

which is the end-to-end BER at the $i$ th $(i=1,2)$ source. 


$$
\begin{aligned}
P_{M, s_{i}}^{k} & =\sum_{\hat{b}_{r_{k}}(n) \in\{-1,1\}} P\left(\hat{b}_{r_{k}, s_{i}}(n)=-1 \mid \hat{b}_{r_{k}}(n), b(n)=1\right) P\left(\hat{b}_{r_{k}}(n) \mid b(n)=1\right) \\
& \stackrel{(a)}{=} \sum_{\hat{b}_{r_{k}}(n) \in\{-1,1\}} P\left(\hat{b}_{r_{k}, s_{i}}(n)=-1 \mid \hat{b}_{r_{k}}(n)\right) P\left(\hat{b}_{r_{k}}(n) \mid b(n)=1\right) \\
& \stackrel{(b)}{=} P_{D, s_{i}}^{k}\left(1-P_{M, r_{k}}\right)+\left(1-P_{D, s_{i}}^{k}\right) P_{M, r_{k}}
\end{aligned}
$$

$$
\eta_{1, k}=\frac{\sigma_{1, k}+\sigma_{2, k}}{4 \gamma \sigma_{1, k} \sigma_{2, k} \min \left(\sigma_{1, k}, \sigma_{2, k}\right)}+\frac{\left(\sigma_{1, k}+\sigma_{2, k}\right)^{2}}{4 \gamma \sigma_{1, k}^{2} \sigma_{2, k}^{2}} \ln \frac{2 \gamma \sigma_{1, k}^{2} \sigma_{2, k}^{2}}{\left(\sigma_{1, k}+\sigma_{2, k}\right)^{2}}
$$

\section{Power Allocation}

Having the closed-form BER (39), we are about to investigate the power allocation among the two sources and the single relay so as to minimize the average system BER, which can be formulated as

$$
\begin{array}{ll}
\min & P_{e}^{k}=\frac{1}{2}\left(P_{e, s_{1}}^{k}+P_{e, s_{2}}^{k}\right) \\
\text { s.t. } & \alpha_{1}+\alpha_{2}+\beta_{k}=1, \\
& 0 \leq \alpha_{1}, \alpha_{2}, \beta_{k} \leq 1 .
\end{array}
$$

However, it is generally hard to directly manipulate the exact BER (39), and the optimal solution can only be derived through exhaustive search. In order to obtain one simple closed-form solution, we choose to examine the asymptotic BER at high SNRs, i.e., $\gamma \gg 1$. After some approximations, we can derive from (26), (29) and (37)

$$
\left\{\begin{array}{l}
P_{M, r_{k}} \approx \frac{c_{M, r_{k}}}{\gamma}, c_{M, r_{k}}=\frac{1}{2 \min \left(\alpha_{1} \sigma_{1, k}^{2}, \alpha_{2} \sigma_{2, k}^{2}\right)} \\
P_{F, r_{k}} \approx \frac{d_{F, r_{k}}}{\gamma} \ln \frac{\gamma}{d_{F, r_{k}}}, d_{F, r_{k}}=\frac{\alpha_{1} \sigma_{1, k}^{2}+\alpha_{2} \sigma_{2, k}^{2}}{2 \alpha_{1} \alpha_{2} \sigma_{1, k}^{2} \sigma_{2, k}^{2}} \\
P_{D, s_{i}}^{k} \approx \frac{q_{D, s_{i}}^{k}}{\gamma}, q_{D, s_{i}}^{k}=\frac{1}{2 \beta_{k} \sigma_{i, k}^{2}}, i=1,2
\end{array}\right.
$$

After plugging these approximations back into (16) and (39), we can obtain the asymptotic average BER at high SNRs, i.e.,

$$
P_{e}^{k} \approx \frac{1}{2 \gamma}\left(c_{M, r_{k}}+d_{F, r_{k}} \ln \frac{\gamma}{d_{F, r_{k}}}+q_{D, s_{1}}^{k}+q_{D, s_{2}}^{k}\right),
$$

where we neglect the higher-order terms. There are several observations here. Firstly, it is easy to see that the BER is dominated by $P_{F, r_{k}}$, which scales as $\gamma^{-1} \ln \gamma$ at high SNRs. Therefore, more power should be allocated to the sources in order to reduce the relay decoding error. Secondly, the BER of the direct transmission with non-coherent DBPSK modulation scales as $\gamma^{-1}$ [23, Eqn.(14-4-28)], which decreases faster than the dominant error term $P_{F, r_{k}}$ at high SNRs. In other words, 2P-DNF-TWR is comparatively not preferred than direct transmission when SNR is increasing, and our simulation results would show this fact later. Finally, it can be observed that $P_{F, r_{k}}>P_{M, r_{k}}$ when source power is fixed and SNR is sufficiently high. This is because it is relatively easier to decode $b(n)$ when the two source symbols have the same signs, in which case the two consecutive observations $y_{k}(n)$ and $y_{k}(n-1)$ would have similar envelopes at high SNRs.

Now let us proceed to solve (40) by use of the asymptotic expression (42). Note that the first two terms in (42) depend only on source power ratio $\alpha_{1}$ and $\alpha_{2}$ while the last two terms only depend on $\beta_{k}$. So the optimization problem (40) can be resolved in two steps. In the first step, we fix $\beta_{k}$ and seek to find the optimal source power, i.e.,

$$
\begin{array}{ll}
\min & \frac{c_{M, r_{k}}+d_{F, r_{k}} \ln \frac{\gamma}{d_{F, r_{k}}}}{2 \gamma} \approx \frac{d_{F, r_{k}}}{2 \gamma} \ln \frac{\gamma}{d_{F, r_{k}}} \\
\text { s.t. } & \alpha_{1}+\alpha_{2}=1-\beta_{k}, \\
& 0 \leq \alpha_{1}, \alpha_{2} \leq 1-\beta_{k} .
\end{array}
$$

where we neglect the term $c_{M, r_{k}}$ because it is much smaller than $\ln \gamma$ at high SNRs. Note that the function $\phi(x)=x \ln x$ is increasing when $x<e^{-1}$, which is the case for sufficiently large $\gamma$. Therefore, it is equivalent to minimizing $d_{F, r_{k}}$ instead in (43), whose optimizer is

$$
\left\{\begin{array}{l}
\alpha_{1}^{o p t}=\left(1-\beta_{k}\right) \frac{\sigma_{2, k}}{\sigma_{1, k}+\sigma_{2, k}} \\
\alpha_{2}^{o p t}=\left(1-\beta_{k}\right) \frac{\sigma_{1, k}}{\sigma_{1, k}+\sigma_{2, k}}
\end{array} .\right.
$$

Clearly, the optimal source power is inversely proportional to the square root of the channel gain of the corresponding source-relay channel. That is, more power should be allocated to the source that is far away from the relay, otherwise its signal would be shadowed by that from the other end during MA phase, which increases the relay decoding error. Therefore, the above source power allocation scheme actually provides an elegant way to resolve the near-far problem. Next, if we plug (44) into (42), it leads to an objective function that only involves the relay power coefficient $\beta_{k}$. After some manipulations, the second optimization problem can be formulated as

$$
\min \frac{\eta_{1, k}}{1-\beta_{k}}+\frac{\eta_{2, k}}{\beta_{k}}, \text { s.t. } 0 \leq \beta_{k} \leq 1,
$$

where $\eta_{1, k}$ is given in (46) on the top of last page and

$$
\eta_{2, k}=\frac{\sigma_{1, k}^{2}+\sigma_{2, k}^{2}}{4 \gamma \sigma_{1, k}^{2} \sigma_{2, k}^{2}} .
$$

Note that we neglect the term $\left(1-\beta_{k}\right)$ within the log function in (46) when deriving the objective function in (45), as it is generally much smaller than $\gamma$ at high SNRs. The optimizer of (45) can be easily derived as

$$
\beta_{k}^{o p t}=\frac{\sqrt{\eta_{2, k}}}{\sqrt{\eta_{1, k}}+\sqrt{\eta_{2, k}}} .
$$

It can be shown that $\beta_{k}^{\text {opt }}$ is a decreasing function of SNR, which coincides with our previous analysis that more power 


$$
\begin{gathered}
P_{e, s_{i}}^{U}=\frac{1}{2}\left\{P\left(\hat{b}_{s_{i}}^{U}(n)=-1 \mid b(n)=1\right)+P\left(\hat{b}_{s_{i}}^{U}(n)=1 \mid b(n)=-1\right)\right\} \\
=\frac{1}{2}\left\{P\left(\left|\bar{D}_{s_{i}}^{U}\right| \geq \frac{K+1}{2} \mid b(n)=1\right)+P\left(\left|D_{s_{i}}^{U}\right| \geq \frac{K+1}{2} \mid b(n)=-1\right)\right\} \\
P\left(\hat{b}_{r_{k}, s_{i}}(n)=-1 \mid b(n)=1\right) \approx P_{M, r_{k}}+P_{D, s_{i}}^{k} \approx \gamma^{-1}\left(c_{M, r_{k}}+q_{D, s_{i}}^{k}\right) \\
P\left(\hat{b}_{r_{k}, s_{i}}(n)=1 \mid b(n)=-1\right) \approx P_{F, r_{k}}+P_{D, s_{i}}^{k} \approx \gamma^{-1}\left(q_{D, s_{i}}^{k}+d_{F, r_{k}} \ln \frac{\gamma}{d_{F, r_{k}}}\right) \\
P\left(\left|\bar{D}_{s_{i}}^{U}\right| \geq \frac{K+1}{2} \mid b(n)=1\right) \approx \gamma^{-\frac{K+1}{2}} \sum_{\left|\bar{D}_{s_{i}}^{U}\right|=\frac{K+1}{2}} \prod_{l \in \bar{D}_{s_{i}}^{U}}\left(q_{D, s_{i}}^{l}+c_{M, r_{l}}\right) \\
P\left(\left|D_{s_{i}}^{U}\right| \geq \frac{K+1}{2} \mid b(n)=-1\right) \approx \gamma^{-\frac{K+1}{2}} \sum_{\left|D_{s_{i}}^{U}\right|=\frac{K+1}{2}} \prod_{m \in D_{s_{i}}^{U}}\left(q_{D, s_{i}}^{m}+d_{F, r_{m}} \ln \frac{\gamma}{d_{F, r_{m}}}\right)
\end{gathered}
$$

should be allocated to the source as SNR is increasing. Another observation is that the power allocation coefficients depend only on the channel gains and system SNR, which are static given the inter-node distances. The relay node can thus estimate these statistics at the very beginning of the transmission by simply measuring the incoming data power, and then feeds back the calculated power allocation coefficients to the two source nodes. As such information exchange is performed only once, the associated overhead is actually negligible.

\section{Performance Analysis: Multi-Relay Case}

In this section, we shall turn our focus to the multi-relay case. However, the exact end-to-end BER analysis based on the ML source decoder (12) is not tractable due to the non-linearity of the decision metric. Alternatively, we seek to characterize the diversity order of the BER performance at high SNRs, which reveals how the system performances improve with the number of relays. Following is the main conclusion of this section.

Proposition: The diversity order of $2 P-D N F-T W R$ with non-coherent DBPSK modulation is

$d(K)=-\lim _{\gamma \rightarrow \infty} \frac{\log P_{e, s_{i}}}{\log \gamma}=\left\{\begin{array}{c}\frac{K+1}{2}, K \text { is odd } \\ \frac{K}{2}, K \text { is even }\end{array}=\left\lceil\frac{K}{2}\right\rceil\right.$,

where $P_{e, s_{i}}$ is the decoding error at the ith $(i=1,2)$ source, and $K$ is the number of relays.

The above result is somewhat counter-intuitive, as the diversity order is only about half of the number of relays. Such performance penalty is due to error propagation, as the relays are assumed to forward whatever they decode without any error correction. To prove this, we seek to find an upper bound and a lower bound on BER and show that they actually share the same diversity order as (49).

\section{A. BER Upper Bound}

In this sub-section, we would derive an upper bound on BER, the diversity order of which provides a lower bound on $d(K)$ in (49). Note that the ML source decoder (12) is optimum in the sense of minimizing the decoding error, thus any suboptimal source decoder would lead to a strictly higher BER. So we simply investigate a post-combining decoder, where the $i$ th $(i=1,2)$ source first applies the single-relay decoder (31) on each relay-source channel and obtains a set of $K$ estimates $\left\{\hat{b}_{r_{k}, s_{i}}(n)\right\}_{k=1}^{K}$, and then feeds these estimates into a combiner whose output is

$$
\hat{b}_{s_{i}}^{U}(n)=\left\{\begin{array}{c}
1, \text { if }\left|D_{s_{i}}^{U}\right|>\left|\bar{D}_{s_{i}}^{U}\right| \\
-1, \text { if }\left|D_{s_{i}}^{U}\right| \leq|\leq| \bar{D}_{s_{i}}^{U} \mid
\end{array},\right.
$$

where $D_{s_{i}}^{U}=\left\{m \mid \hat{b}_{r_{m}, s_{i}}(n)=1\right\}$ with the complement set being $\bar{D}_{s_{i}}^{U}$. Now we shall analyze the BER of this decoder at high SNRs.

When $K$ is odd, the decision rule (50) is equivalent to $\hat{b}_{s_{i}}^{U}(n)=1$ if $\left|D_{s_{i}}^{U}\right| \geq \frac{K+1}{2}$. So the decoding error at the $i$ th $(i=1,2)$ source can be written in a similar way as (33), which is given in (51) on the top of this page. Note that the decodings on different relay-source channels are independent conditioned on $b(n)$, and the conditional decoding errors at high SNRs for the $k$ th $(k=1,2, \ldots, K)$ branch can be derived from (36), (38) and (41) as given in (52) and (53) on the top of last page. Therefore, we have (54) and (55) on the top of last page, where we neglect the higher-order terms of $\gamma^{-1}$. Clearly, $P_{e, s_{i}}^{U}$ has a diversity order of $\frac{K+1}{2}$ in this case as both of the two components have the same diversity orders.

The case when $K$ is even can be characterized in a similar way. Now the decision rule (50) is reduced to $\hat{b}_{s_{i}}^{U}(n)=1$ if $\left|D_{s_{i}}^{U}\right| \geq \frac{K}{2}+1$, and the decoding error is given in (56) on the top of this page. From (52) and (53), we can obtain the two kinds of conditional decoding error at high SNRs as (57) and (58) given on the top of this page. As the decoding error (56) is dominated by (57), its diversity order is actually $\frac{K}{2}$. Combining these two cases would lead to the diversity order $d(K)$ given in (49).

\section{B. BER Lower Bound}

In this sub-section, we would instead derive a lower bound on BER, the diversity order of which provides an upper bound 


$$
\begin{gathered}
P_{e, s_{i}}^{U}=\frac{1}{2}\left\{P\left(\left|\bar{D}_{s_{i}}^{U}\right| \geq \frac{K}{2} \mid b(n)=1\right)+P\left(\left|D_{s_{i}}^{U}\right| \geq \frac{K}{2}+1 \mid b(n)=-1\right)\right\} \\
P\left(\left|\bar{D}_{s_{i}}^{U}\right| \geq \frac{K}{2} \mid b(n)=1\right) \approx \gamma^{-\frac{K}{2}} \sum_{\left|\bar{D}_{s_{i}}^{U}\right|=\frac{K}{2}} \prod_{l \in \bar{D}_{s_{i}}^{U}}\left(q_{D, s_{i}}^{l}+c_{M, r_{l}}\right) \\
P\left(\left|D_{s_{i}}^{U}\right| \geq \frac{K}{2}+1 \mid b(n)=-1\right) \approx \gamma^{-\left(\frac{K}{2}+1\right)} \sum_{\left|D_{s_{i}}^{U}\right|=\frac{K}{2}+1} \prod_{m \in D_{s_{i}}^{U}}\left(q_{D, s_{i}}^{m}+d_{F, r_{m}} \ln \frac{\gamma}{d_{F, r_{m}}}\right)
\end{gathered}
$$

$$
\begin{gathered}
P_{e, s_{i}}^{U}=\frac{1}{2}\left\{P\left(\left|\bar{D}_{s_{i}}^{L}\right| \geq \frac{K+1}{2} \mid b(n)=1\right)+P\left(\left|D_{s_{i}}^{L}\right| \geq \frac{K+1}{2} \mid b(n)=-1\right)\right\} \\
P\left(\left|\bar{D}_{s_{i}}^{L}\right| \geq \frac{K+1}{2} \mid b(n)=1\right) \approx \gamma^{-\frac{K+1}{2}}\left(\begin{array}{c}
K \\
\frac{K+1}{2}
\end{array}\right) c_{M}^{\frac{K+1}{2}} \\
P\left(\left|D_{s_{i}}^{L}\right| \geq \frac{K+1}{2} \mid b(n)=-1\right) \approx \gamma^{-\frac{K+1}{2}}\left(\begin{array}{c}
K \\
\frac{K+1}{2}
\end{array}\right)\left(d_{F} \ln \frac{\gamma}{d_{F}}\right)^{\frac{K+1}{2}}
\end{gathered}
$$

$$
\begin{aligned}
& P_{e, s_{i}}^{L}=\frac{1}{2}\left\{P\left(\left|\bar{D}_{s_{i}}^{L}\right| \geq \frac{K}{2} \mid b(n)=1\right)+P\left(\left|D_{s_{i}}^{L}\right| \geq \frac{K}{2}+1 \mid b(n)=-1\right)\right\} \\
& P\left(\left|\bar{D}_{s_{i}}^{L}\right| \geq \frac{K}{2} \mid b(n)=1\right) \approx \gamma^{-\frac{K}{2}}\left(\begin{array}{c}
K \\
\frac{K}{2}
\end{array}\right) c_{M}^{\frac{K}{2}} \\
& P\left(\left|D_{s_{i}}^{L}\right| \geq \frac{K}{2}+1 \mid b(n)=-1\right) \approx \gamma^{-\left(\frac{K}{2}+1\right)}\left(\begin{array}{c}
K \\
\frac{K}{2}+1
\end{array}\right)\left(d_{F} \ln \frac{\gamma}{d_{F}}\right)^{\frac{K}{2}+1}
\end{aligned}
$$

on $d(K)$ in (49). Here we use a similar technique proposed in [14]. Specifically, we shall make the following two ideal assumptions, i.e.,

(1) The relay-source channel is distortion free, i.e., $r_{k, i}(n)=t_{k}(n)$, such that both sources can know the decoded symbols $\left\{\hat{b}_{r_{k}}(n)\right\}_{k=1}^{K}$ at all relays through differential demodulation;

(2) All relays have the same decoding ability as the best relay, i.e., $P_{M}=\min _{k \in\{1,2, \ldots K\}} P_{M, r_{k}} \stackrel{\gamma \gg 1}{\approx} \frac{c_{M}}{\gamma}$ where $c_{M}=$ $\min _{k \in\{1,2, \ldots K\}} c_{M, r_{k}}$, and $P_{F}=\min _{k \in\{1,2, \ldots K\}} P_{F, r_{k}} \stackrel{\gamma \gg 1}{\approx} \frac{d_{F}}{\gamma} \ln \frac{\gamma}{d_{F}}$ where $d_{F}=\min _{k \in\{1,2, \ldots K\}} d_{F, r_{k}}$.

Note that both of these two assumptions bring positive contributions to system performances, therefore helping to lower the BER. Like (9), the single-symbol ML decoder at the $i$ th $(i=1,2)$ source can be written as

$$
\begin{aligned}
\hat{b}_{s_{i}}^{L}(n) & =\arg \max _{b(n) \in\{-1,1\}} f\left(\left\{\hat{b}_{r_{k}}(n)\right\}_{k=1}^{K} \mid b(n)\right) \\
& =\operatorname{sign}\left(\left|D_{s_{i}}^{L}\right| \ln \frac{1-P_{M}}{P_{F}}+\left|\bar{D}_{s_{i}}^{L}\right| \ln \frac{P_{M}}{1-P_{F}}\right)(59)
\end{aligned}
$$

where $D_{s_{i}}^{L}=\left\{m \mid \hat{b}_{r_{m}}(n)=1\right\}$ with the complement set being $\bar{D}_{s_{i}}^{L}$. At high SNRs, both $P_{M}$ and $P_{F}$ approach 0 and $\frac{\ln P_{M}}{\ln P_{F}} \stackrel{\gamma \gg 1}{\approx} 1$, so the above decision rule is reduced to

$$
\hat{b}_{s_{i}}^{L}(n)=\left\{\begin{array}{c}
1, \text { if }\left|D_{s_{s}}^{L}\right|>\left|\bar{D}_{s_{i}}^{L}\right| \\
-1, \text { if }\left|D_{s_{i}}^{L}\right| \leq\left|\bar{D}_{s_{i}}^{L}\right|
\end{array},\right.
$$

which is similar to (50). So the error analysis can be done in the same way as we did in the last sub-section, and we shall skip some tedious intermediate steps and directly give the final results. When $K$ is odd, the decoding error at the $i$ th $(i=1,2)$ source at high SNRs is given in (61)-(63) on the top of this page. Otherwise when $K$ is even, the decoding error at the $i$ th $(i=1,2)$ source at high SNRs is given in (64)-(66) on the top of this page. Comparing (51)-(58) with (61)-(66), we can observe that the two BER bounds have exactly the same diversity order as (49), thus completing the proof.

\section{Numerical Results}

In this section, we present simulation results for 2PDNF-TWR system using non-coherent DBPSK modulation. Throughout our simulations, we use the path loss model $\sigma^{2}=d^{-4}$, where $\sigma^{2}$ is the channel gain and $d$ is the distance between two terminals. For simplicity, we normalize the distance between two sources to 1, and we always place the relays on the line connecting two sources. In all cases, BER refers to the average decoding error at source 1 and source 2. Without special explanation, the transmit power is always equally split among all terminals.

We first examine the performance of a single-relay system, where $d_{1, r}$ and $d_{2, r}$ are the distances between the relay and two sources, respectively. In Fig. 2, we compare the BER of different relay decoders with the theoretical results. The suboptimal relay decoder refers to the MUD followed by a PLNC encoder. It can be observed that there is almost no difference between the ML decoder and the suboptimal one, and both of them coincide with the theoretical results given 


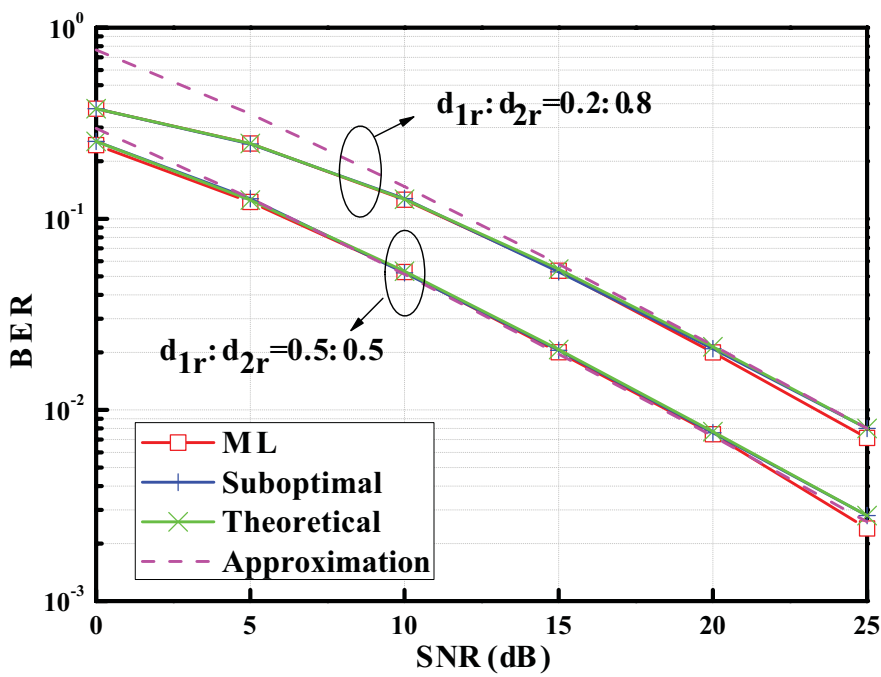

Fig. 2. BER performances versus SNR.

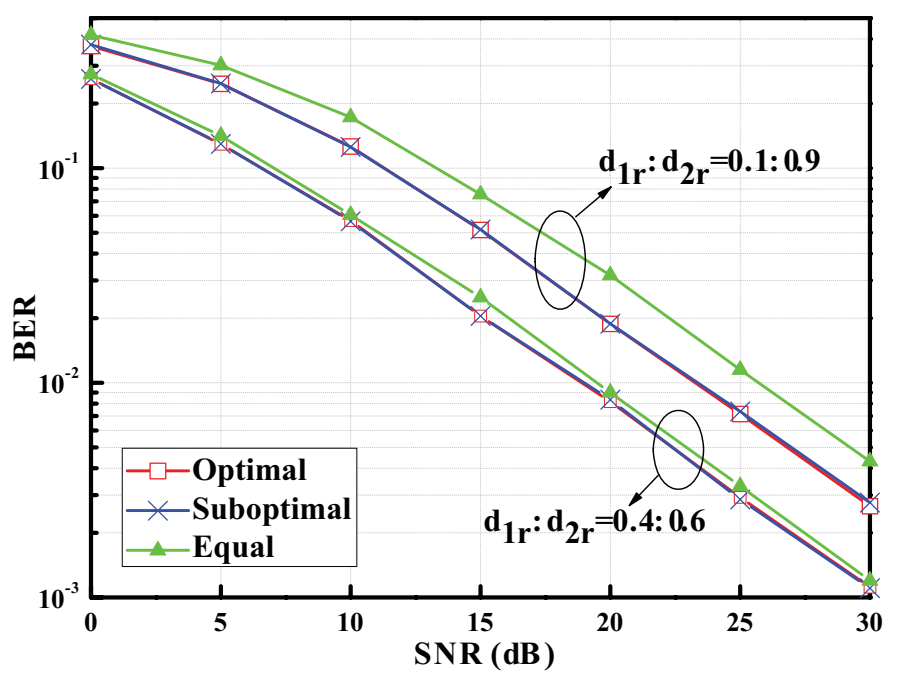

Fig. 3. BER performances with power allocation versus SNR.

by (39). Besides, the asymptotic BER (42) is tight when SNR is sufficiently high, e.g., when $\gamma \geq 15 d B$ for $d_{1, r}: d_{2, r}=0.2: 0.8$ and when $\gamma \geq 5 d B$ for $d_{1, r}: d_{2, r}=0.5: 0.5$. The tightness for the latter case is due to the high channel gains of both the sourcerelay channels, which make it easier to satisfy the high SNR assumption.

Then in Fig. 3 and Fig. 4, we proceed to study the benefits of power allocation. The optimal scheme is found through exhaustive search, and the suboptimal one refers to that given by (44) and (48) derived through asymptotic analysis. As we can see, the suboptimal scheme performs almost as well as the optimal scheme in most cases. From Fig. 4, we can observe some slight performance degradation when the SNR is low and the relay is far from source 2 . This is because the channel SNR from source 2 to the relay is so low that the high SNR assumption is not fully effective on that channel. Compared with equal power allocation, about $2 \mathrm{~dB}$ SNR gain can be observed in Fig. 3 when $d_{1, r}: d_{2, r}=0.1: 0.9$. Such performance gain is diminishing as the relay moves to the halfway between two sources, in which case the equal power allocation is nearoptimal.

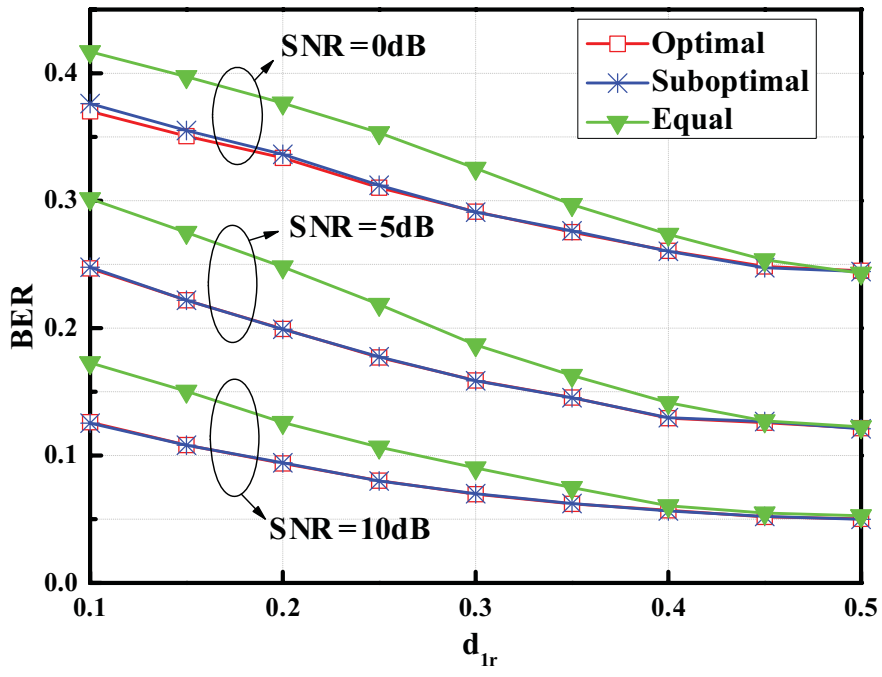

Fig. 4. BER performances with power allocation versus relay placement.

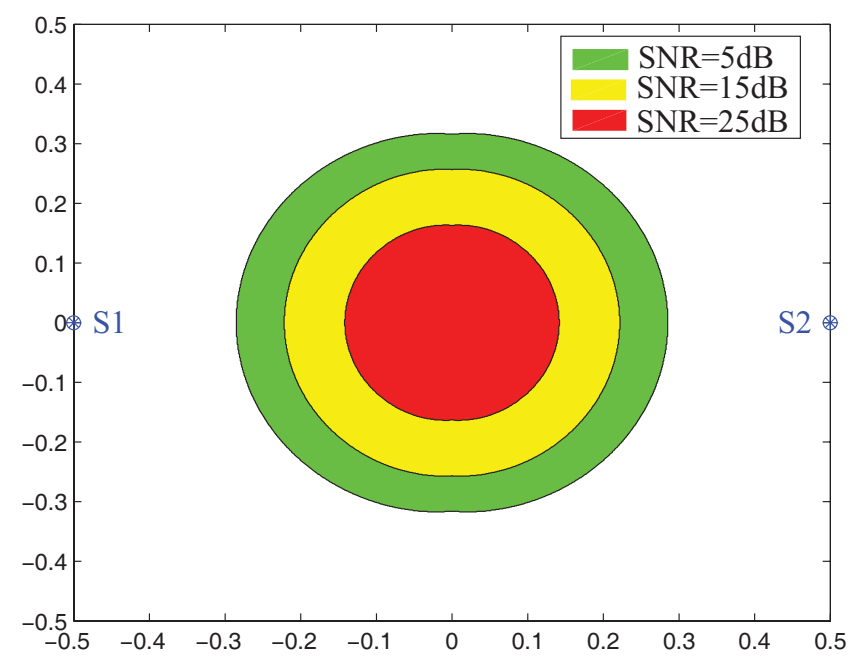

Fig. 5. Comparison of 2P-DNF-TWR and direct transmission. Colored areas correspond to where 2P-DNF-TWR can achieve lower BER.

We also compare the 2P-DNF-TWR with direct transmission using the same modulation scheme in Fig. 5. To do this, we locate the two sources at $(-0.5,0)$ and $(0.5,0)$, respectively. We then compare the BER of these two systems at each grid on a square plane, and the colored areas correspond to where 2P-DNF-TWR can achieve lower BER. To fairly compare the performances, we split the power equally between two sources for the direct transmission; as for the 2P-DNF-TWR, we use a mixed power allocation scheme that first determines the source power ratio by (44) and then finds the optimal relay power through one-dimensional search so as to reduce the time complexity. As we can see, the preferred relay locations are always concentrated around the halfway between two sources, otherwise the 2P-DNF-TWR cannot benefit from the high channel gains resulted from the shorter source-relay distances. Another observation is that the preferred relay locations actually shrink as SNR is increasing. This coincides with our analysis in Section III.C that direct transmission is more preferred at high SNRs.

Finally in Fig. 6 and Fig. 7 we investigate the multi-relay 


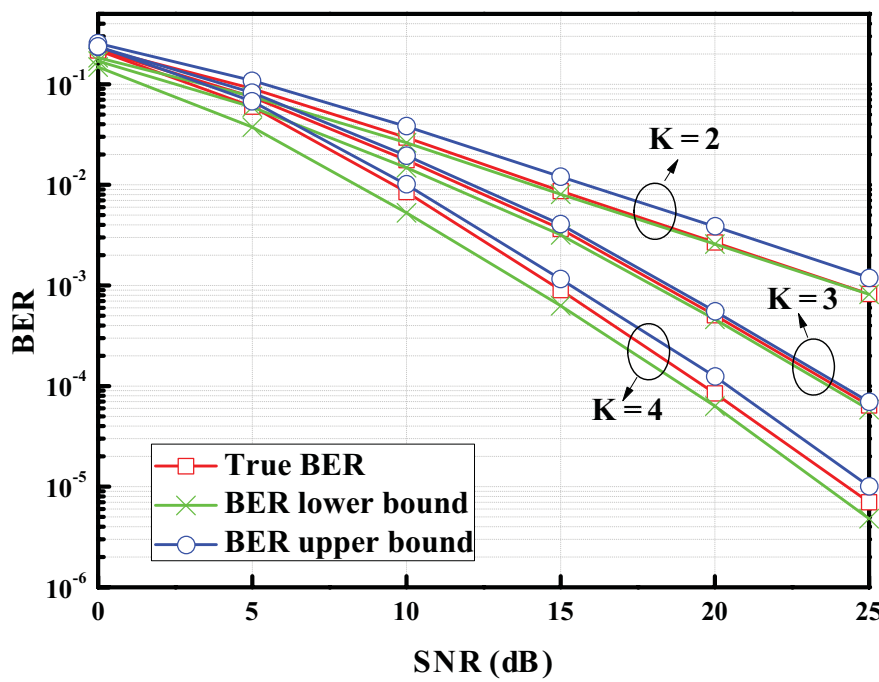

Fig. 6. BER performances with multiple relays - all relays are at halfway between two sources.

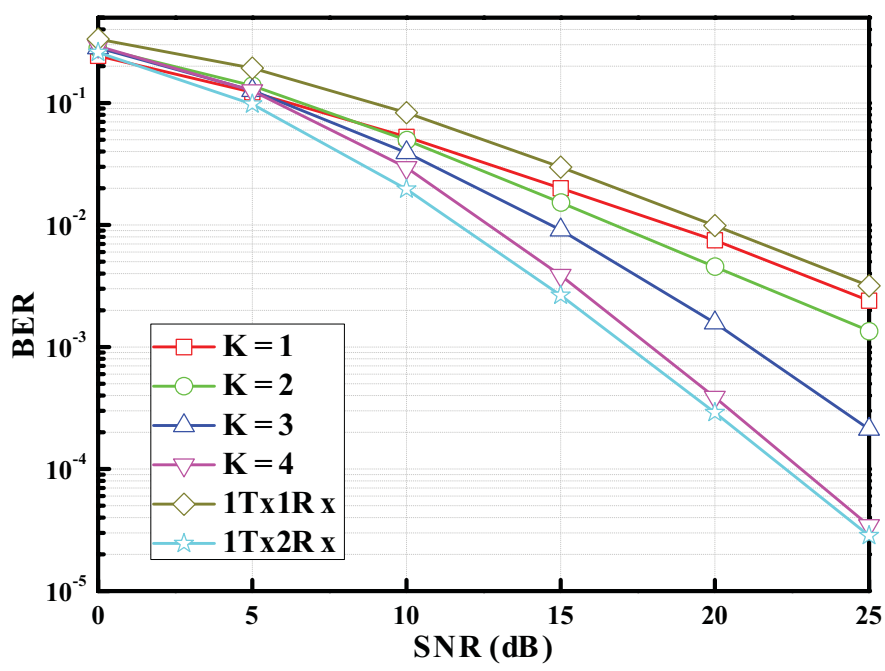

Fig. 7. BER performances with multiple relays - all relays are equispaced between two sources.

scenario. We first locate all relays at halfway between two sources, in which case they should have the same decoding ability. As we can see from Fig. 6, both of the BER bounds are tight in all cases, and they have the same slopes as we showed before. In Fig. 7, we further compare 2P-DNF-TWR with the typical receive diversity system using one transmit antenna and $K$ receive antenna (1Tx $K \mathrm{Rx})$, which is well known to have a diversity order of $K$ [23, Eqn.(14-4-28)]. It is clear that the diversity order of the system having 1 relay or 2 relays is 1 as $1 \mathrm{Tx} 1 \mathrm{Rx}$, and the system having 3 relays or 4 relays has a diversity order of 2 as $1 \mathrm{Tx} 2 \mathrm{Rx}$, which validates our proposition (49). It should be mentioned that as all relays operate on orthogonal channels, adding more relays would reduce the spectral efficiency. Since the diversity gain is achieved at a double loss of spectral efficiency, it is better to deploy only a small number of relays in practical systems so as to trade off these two performance measures.

\section{CONCLUSION AND Future WORK}

In this work, we have developed ML decoders for $2 \mathrm{P}$ DNF-TWR system using non-coherent DBPSK modulation and analyzed the corresponding error performances. For the single-relay case, the closed-form BER is obtained after approximating the ML relay decoder as the MUD followed by a PLNC encoder, and a near-optimal power allocation is derived based on asymptotic analysis at high SNRs. For the multirelay case with $K$ parallel relays, though the exact analysis is intractable, we prove that the diversity order is exactly $\left\lceil\frac{K}{2}\right\rceil$ by developing proper bounds on BER performances. Future work may focus on denoising function design of 2P-DNFTWR system using higher-order non-coherent modulations, which is still an open problem. One may also investigate the selective relaying protocol to recover the full diversity order.

\section{REFERENCES}

[1] R. Pabst, B. H. Walke, D. C. Schultz, P. Herhold, H. Yanikomeroglu, S. Mukherjee, H. Viswanathan, M. Lott, W. Zirwas, M. Dohler, H. Aghvami, D. D. Falconer, and G. P. Fettweis, "Relay-based deployment concepts for wireless and mobile broadband radio," IEEE Commun. Mag., vol. 42, no. 9, pp. 80-89, Sep. 2004.

[2] J. N. Laneman, D. N. C. Tse, and G. W. Wornell, "Cooperative diversity in wireless networks: efficient protocols and outage behavior," IEEE Trans. Inf. Theory, vol. 50, no. 12, pp. 3062-3080, Dec. 2004.

[3] K. J. R. Liu, A. K. Sadek, W. Su, and A. Kwasinski, Cooperative Communications and Networking. Cambridge University Press, 2008.

[4] B. Rankov and A. Wittneben, "Spectral efficient protocols for halfduplex fading relay channels," IEEE J. Sel. Areas Commun., vol. 25, no. 2, pp. 379-389, Feb. 2007

[5] A. S. Ibrahim, A. K. Sadek, W. Su, and K. J. R. Liu, "Cooperative communications with relay selection: when to cooperate and whom to cooperate with?" IEEE Trans. Wireless Commun., vol. 7, no. 7, pp. 2814-2827, July 2008.

[6] P. Larsson, N. Johansson, and K.-E. Sunell, "Coded bi-directional relaying," in Proc. IEEE VTC-Spring, vol. 2, pp. 851-855, May 2006.

[7] R. Ahlswede, N. Cai, S.-Y. R. Li, and R. W. Yeung, "Network information flow," IEEE Trans. Inf. Theory, vol. 46, no. 4, pp. 1204-1216, July 2000.

[8] R. H. Y. Louie, Y. H. Li, and B. Vucetic, "Practical physical layer network coding for two-way relay channels: performance analysis and comparison," IEEE Trans. Wireless Commun., vol. 9, no. 2, pp. 764-777, Feb. 2010.

[9] P. Popovski and H. Yomo, "The anti-packets can increase the achievable throughput of a wireless multi-hop network," in Proc. IEEE International Conference on Communication, pp. 3885-3890, June 2006.

[10] S. Zhang, S. C. Liew, and P. P. Lam, "Physical-layer network coding," in ACM MOBICOM, Los Angeles, Sep. 2006.

[11] P. Popovski and H. Yomo, "Physical network coding in two-way wireless relay channels," in Proc. IEEE International Conference on Communication, pp. 707-712, June 2007.

[12] E. C. Y. Peh, Y.-C. Liang, and Y. L. Guan, "Power control for physicallayer network coding in fading environments," in Proc. IEEE Personal, Indoor and Mobile Radio Commun., pp. 1-5, Sep. 2008.

[13] P. Popovski and H. Yomo, "Wireless network coding by amplify-andforward for bi-directional traffic flows," IEEE Commun. Lett., vol. 11, no. 1, pp. 16-19, Jan. 2007.

[14] D. Chen and J. N. Laneman, "Modulation and demodulation for cooperative diversity in wireless systems," IEEE Trans. Wireless Commun., vol. 5, no. 7, pp. 1785-1794, July 2006.

[15] T. Himsoon, W. P. Siriwongpairat, W. F. Su, and K. J. R. Liu, "Differential modulation with threshold-based decision combining for cooperative communications," IEEE Trans. Signal Process., vol. 55, no. 7, pp. 3905-3923, July 2007.

[16] T. Himsoon, W. P. Siriwongpairat, W. Su, and K. J. R. Liu, "Differential modulation for multinode cooperative communications," IEEE Trans. Signal Process., vol. 56, no. 7, pp. 2941-2956, July 2008.

[17] J. H. Yuan, Y. H. Li, and L. Chu, "Differential modulation and relay selection with detect-and-forward cooperative relaying," IEEE Trans. Veh. Technol., vol. 59, no. 1, pp. 261-268, Jan. 2010. 
[18] Y. L. Zhu, P. Y. Kam, and Y. Xin, "Differential modulation for decodeand-forward multiple relay systems," IEEE Trans. Commun., vol. 58, no. 1, pp. 189-198, Jan. 2010.

[19] S. Katti, S. Gollakota, and D. Katabi, "Embracing wireless interference: analog network coding," in ACM SIGCOMM 2007, Aug. 2007.

[20] L. Y. Song, G. Hong, B. L. Jiao, and M. Debbah, "Joint relay selection and analog network coding using differential modulation in two-way relay channels," IEEE Trans. Veh. Technol., vol. 59, no. 6, pp. 29322939, July 2010.

[21] T. Cui, F. F. Gao, and C. Tellambura, "Differential modulation for two-way wireless communications: a perspective of differential network coding at the physical layer," IEEE Trans. Commun., vol. 57, no. 10, pp. 2977-2987, Oct. 2009.

[22] T. Koike-Akino, P. Popovski, and V. Tarokh, "Optimized constellations for two-way wireless relaying with physical network coding," IEEE J. Sel. Areas Commun., vol. 27, no. 5, pp. 773-787, June 2009.

[23] J. Proakis, Digital Communications, 4th edition. McGraw-Hill, 2001.

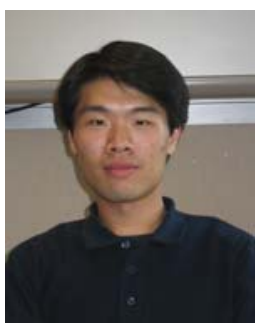

Wei Guan received the B.S. in Electrical Engineering and Finance (double degree) in 2006, and M.S. (with highest honor) in Electrical Engineering in 2009, both from Shanghai JiaoTong University, Shanghai, China. Now he is a Ph.D. student in the Department of Electrical and Computer Engineering at University of Maryland, College Park.

His current research interests are in the areas of wireless communications and networks, including cooperative communications and network coding. $\mathrm{He}$ received the 1st Prize in the 18th National Physics Contest, Shanghai, and the A. James Clark School of Engineering Distinguished Graduate Fellowship from University of Maryland, College Park in 2009.

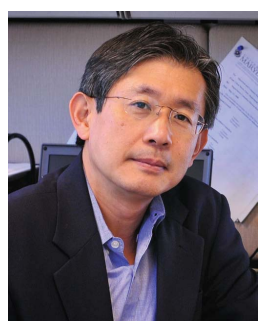

K. J. Ray Liu (F'03) is named a Distinguished Scholar-Teacher of University of Maryland, College Park, in 2007, where he is Christine Kim Eminent Professor of Information Technology. He serves as Associate Chair of Graduate Studies and Research of Electrical and Computer Engineering Department and leads the Maryland Signals and Information Group conducting research encompassing broad aspects of wireless communications and networking, information forensics and security, multimedia signal processing, and biomedical engineering.

Dr. Liu is the recipient of numerous honors and awards including IEEE Signal Processing Society Technical Achievement Award and Distinguished Lecturer. He also received various teaching and research recognitions from University of Maryland including university-level Invention of the Year Award; and Poole and Kent Senior Faculty Teaching Award and Outstanding Faculty Research Award, both from A. James Clark School of Engineering. An ISI Highly Cited Author in Computer Science, Dr. Liu is a Fellow of IEEE and AAAS.

Dr. Liu is President-Elect and was Vice President - Publications of IEEE Signal Processing Society. He was the Editor-in-Chief of IEEE Signal Processing Magazine and the founding Editor-in-Chief of EURASIP Journal on Advances in Signal Processing. 